\title{
Fast Model Predictive Control on a Smartphone-based Flight Controller
}

\author{
Luis Garcia \\ Industrial Control Research Group \\ Universidad del Valle \\ Cali, Colombia \\ luis.linares@correounivalle.edu.co
}

\author{
Alejandro Astudillo \\ MECO Research Team, KU Leuven \\ DMMS Lab, Flanders Make \\ Leuven, Belgium \\ alejandro.astudillovigoya@kuleuven.be
}

\author{
Esteban Rosero \\ Industrial Control Research Group \\ Universidad del Valle \\ Cali, Colombia \\ esteban.rosero@correounivalle.edu.co
}

\begin{abstract}
Several attempts have been made to execute control of a system using only a smartphone's processor running computationally inexpensive algorithms such as PID, LQR or $H_{\infty}$ controllers. This paper presents design and implementation of model predictive controllers on a smartphone using the numerical optimization framework CasADi. To evaluate this framework's performance (and compare its results with those from a Java library JOptimizer's deployment) the implemented model predictive control algorithm was subjected to simulations of running a quadrotor control system on a smartphone. It attained a tracking error of $0.0693 \mathrm{~m}$. These evaluation results open the possibility of implementing more computationally expensive algorithms on a smartphone's processor including online or real-time usage.
\end{abstract}

Index Terms-optimization, predictive control, quadrotor, smartphone, trajectory tracking.

\section{INTRODUCTION}

Every year, off-the-shelf smartphones are enriched with new features giving them additional computational power and instrumentation capabilities. These features allow the user to execute computationally expensive algorithms in the smartphone [1], integrating large amount of data coming from the sensors embedded in it. Therefore, smartphones have attracted the attention of researchers who seek to exploit such features.

Although some researchers tend to disregard their capabilities, smartphones can significantly contribute to control applications in the role of main computing and sensing unit. Some attempts have been made to use a smartphone as computation unit for a control system. In [2], a soccer robot agent based on an Android smartphone was developed. A smartphone-based navigation algorithm, combining PID based depth control system and line tracking, was used to manage a micro-sized autonomous underwater vehicle in [3]. In [4], a motion planning application and a quadrotor control, including visual odometry for mapping, was developed. Moreover, optimal and robust controllers, and a state estimator based on a Kalman filter for a smartphone-based quadrotor were described in [5], while in [6] an attitude and altitude cascade controller for a smartphone-based quadrotor was exposed.

Luis Garcia and Alejandro Astudillo contributed equally to this work.
A Model Predictive Controller (MPC) is designed based on Optimal Control Problem (OCP) which is solved at every sample time, following a receding horizon fashion [7]. The OCP minimizes an objective function subject to constraints, e.g., system dynamics, actuation limits, initial and final state constraints, among others. After solving the OCP with some uncertainty, the control input corresponding to the first prediction is applied to the system, and the OCP solution algorithm starts again [8], [9]. In MPC, the control law is required to be calculated as fast as possible to enable an online implementation. This is a major concern in embedded implementations of MPCs. For a smartphone to be capable of taking the role as a computation unit of a MPC system, it should be able to solve the OCP in less than the sample time.

Smartphone applications rely on numerical solvers to solve OCPs. If using the Android operating system, such solvers can be written in Java or C++ language. JOptimizer is an open source Java library that aims to solve convex optimization problems [10]. This library has been used in several projects, such as [11] where it was used for solving quadratic optimization problems in Markov decision processes, or [12], where it was used to compute the control law of a model predictive controller for a harbor defense scenario.

On the other hand, CasADi is an open-source framework for numerical optimization writen in C++ [13]. It can be interfaced from MATLAB and Python, or used directly using $\mathrm{C}++$. This framework has been widely use in research. In [14] it was used to compute optimal trajectories for robot manipulators. A nonlinear MPC for a human-sized quadrotor was designed and implemented using CasADi [15]. However, to the best of the authors' knowledge, there has not been any exploration about using CasADi to ease the implementation of control systems in smartphones.

After implementing a linear MPC in Android using the Java library JOptimizer [16], now we extend the possibilities of control algorithm implementation in smartphones with the use of CasADi code-generation capabilities and the Java Native Interface (JNI).

This paper presents the implementation of a trajectorytracking MPC algorithm on an Android smartphone. The optimal control problem is solved using of both the JOptimizer 


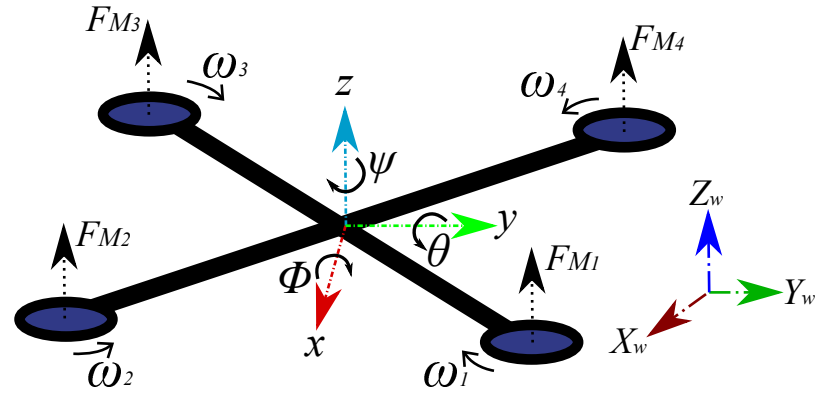

Fig. 1. Quadrotor squeme in ' $\mathrm{X}$ ' configuration.

library and the CasADi framework. The trajectory-tracking MPC is solved taking into account the dynamics of a quadrotor.

The rest of the paper is structured as follows. The dynamic model of the quadrotor in X-configuration is presented in Section II. In Section III, the description of a linear MPC strategy for trajectory-tracking is shown. The results of the linear MPC implementation in the smartphone are described in Section IV. A nonlinear MPC being solved in the smartphone is presented in Section V. Finally, some conclusion remarks are presented in Section VI.

\section{QUADROTOR's MODEL}

The quadrotor is modeled as a symmetrical rigid body with six degrees of freedom (DoF), which compose the quadrotor pose vector

$$
\boldsymbol{\Gamma}=\left[\begin{array}{llllll}
x & y & z & \psi & \theta & \phi
\end{array}\right]^{T},
$$

where $(x, y, z)$ describe the quadrotor's position with respect to its body-frame, and $(\psi, \theta, \phi)$ represents the angular deviation between the body-frame and the Earth-frame shown as $\left(X_{W}, Y_{W}, Z_{W}\right)$ in Fig. 1.

Describing the dynamics based on the variation of linear and angular momenta on the rigid body, and using the quadrotor geometry shown in Fig. 1, the quadrotor nonlinear model is obtained using the Newton-Euler approach as follows.

The model is based on a state vector

$$
\mathbf{x}=\left[\begin{array}{llllllllllll}
x & \dot{x} & y & \dot{y} & z & \dot{z} & \psi & \dot{\psi} & \theta & \dot{\theta} & \phi & \dot{\phi}
\end{array}\right]^{T},
$$

and an input vector

$$
\mathbf{u}=\left[\begin{array}{llll}
u_{1} & u_{2} & u_{3} & u_{4}
\end{array}\right]^{T}=\left[\begin{array}{llll}
T_{u} & \tau_{\psi} & \tau_{\theta} & \tau_{\phi}
\end{array}\right]^{T},
$$

where $(\cdot)$ represents the derivative of a variable with respect to time. The quadrotor inputs are defined as $T_{u}$ the total thrust exerted by the motors in the $z$-direction, and $\left(\tau_{\psi}, \tau_{\theta}, \tau_{\phi}\right)$ the torques applied by the motors about the $z, y$ and $x$ axes respectively. These inputs are linear combinations of the motor forces $\left(F_{M_{i}}, i \in\{1,2,3,4\}\right)$, and are defined as

$$
\mathbf{u}=\left[\begin{array}{cccc}
1 & 1 & 1 & 1 \\
-k_{M} & k_{M} & -k_{M} & k_{M} \\
-L_{X} & -L_{X} & L_{X} & L_{X} \\
-L_{X} & L_{X} & L_{X} & -L_{X}
\end{array}\right]\left[\begin{array}{c}
F_{M_{1}} \\
F_{M_{2}} \\
F_{M_{3}} \\
F_{M_{4}}
\end{array}\right] .
$$

where $k_{M}$ is a proportional constant, and $L_{X}=L \cos (\pi / 4)$ with $L$ being the distance between the origin of the quadrotor's body frame and the center of a motor [17].

The quadrotor dynamics are not only affected by $\mathbf{u}$, but also by other forces and torques such as those generated by gravity

$$
\mathbf{G}_{\boldsymbol{\Lambda}}=m g\left[\begin{array}{c}
-\sin (\theta) \\
-\cos (\theta) \sin (\phi) \\
\cos (\theta) \cos (\phi) \\
\mathbf{0}_{\mathbf{3} \times \mathbf{1}}
\end{array}\right],
$$

by the wind $\mathbf{W}_{\boldsymbol{\Lambda}} \in \mathbb{R}^{6 \times 1}$, and the gyroscopic effects $\mathbf{P}_{\boldsymbol{\Lambda}} \in$ $\mathbb{R}^{6 \times 1}$ produced by the rotation of the motor propellers [18]. For the sake of simplicity $\mathbf{W}_{\boldsymbol{\Lambda}}$ and $\mathbf{P}_{\boldsymbol{\Lambda}}$ contributions are assumed as disturbances and are not taken into account in the model.

Let us define the augmented input vector

$$
\mathbf{u}_{\boldsymbol{\Lambda}}=\left[\begin{array}{llllll}
0 & 0 & T_{u} & \tau_{\psi} & \tau_{\theta} & \tau_{\phi}
\end{array}\right]^{T},
$$

and quadrotor nonlinear dynamics

$$
\mathbf{0}_{\mathbf{6} \times \mathbf{1}}=\mathbf{M}_{\boldsymbol{\Lambda}}{ }^{-1}\left(-\mathbf{S}_{\boldsymbol{\Lambda}}-\mathbf{G}_{\boldsymbol{\Lambda}}+\mathbf{u}_{\boldsymbol{\Lambda}}\right),
$$

with

$$
\begin{aligned}
\mathbf{M}_{\boldsymbol{\Lambda}}= & \operatorname{diag}\left(m, m, m, J_{z z}, J_{y y}, J_{x x}\right), \\
\mathbf{S}_{\boldsymbol{\Lambda}}= & {\left[\begin{array}{c}
m(\ddot{x}+\dot{z} \dot{\theta}-\dot{y} \dot{\phi}) \\
m(\ddot{y}+\dot{x} \dot{\phi}-\dot{z} \dot{\psi}) \\
m(\ddot{z}+\dot{y} \dot{\psi}-\dot{x} \dot{\theta}) \\
J_{z z} \ddot{\psi}+\dot{\theta} \dot{\phi}\left(J_{x x}-J_{y y}\right) \\
J_{y y} \ddot{\theta}+\dot{\psi} \dot{\phi}\left(J_{z z}-J_{x x}\right) \\
J_{x x} \ddot{\phi}+\dot{\psi} \dot{\theta}\left(J_{y y}-J_{z z}\right)
\end{array}\right], }
\end{aligned}
$$

where $m \in \mathbb{R}$ is the quadrotor's mass (in $\mathrm{kg}$ ), $J_{z z}, J_{y y}, J_{x x} \in$ $\mathbb{R}$ are the moments of inertia about the quadrotor's body-frame (in $k g \cdot m^{2}$ ), and $\operatorname{diag}\left(v_{1}, \cdots, v_{n}\right) \in \mathbb{R}^{n \times n}$ is a diagonal matrix with diagonal entries $v_{1}, \cdots, v_{n}$.

Solving (7) for $\ddot{\boldsymbol{\Gamma}}$, and recalling the state and input vectors $(\mathbf{x}, \mathbf{u})$, the dynamics can be written as

$$
f(\mathbf{x}, \mathbf{u})=\dot{\mathbf{x}}=\left[\begin{array}{c}
\dot{x} \\
\dot{y} \dot{\phi}-\dot{z} \dot{\theta}+g \sin (\theta) \\
\dot{y} \\
\dot{z} \dot{\psi}-\dot{x} \dot{\phi}+g \cos (\theta) \sin (\phi) \\
\dot{z} \\
\dot{x} \dot{\theta}-\dot{y} \dot{\psi}-g \cos (\theta) \cos (\phi)+\frac{T_{u}}{m} \\
\dot{\psi} \\
\dot{\phi} \dot{\theta} \frac{J_{x x}-J_{y y}}{J_{z z}}+\frac{\tau_{\psi}}{J_{z z}} \\
\dot{\phi} \dot{\psi} \frac{J_{z z}-J_{x x}}{J_{y y}}+\frac{\tau_{\theta}}{J_{y y}} \\
\dot{\phi} \dot{\psi} \frac{J_{y y}-J_{z z}}{J_{x x}}+\frac{\tau_{\phi}}{J_{x x}}
\end{array}\right] .
$$

These nonlinear dynamics can, however, be linearized with methods such as Jacobian linearization. Thus, the nonlinear 
model in (10) is approximated to a linear state-space representation as

$$
\begin{aligned}
f(\mathbf{x}, \mathbf{u}) & \approx A \mathbf{x}+B \mathbf{u}, \\
A & =\left.\frac{\partial f(\mathbf{x}, \mathbf{u})}{\partial \mathbf{x}}\right|_{\substack{\mathbf{x}=\overline{\mathbf{x}} \\
\mathbf{u}=\overline{\mathbf{u}}}}, \\
B & =\left.\frac{\partial f(\mathbf{x}, \mathbf{u})}{\partial \mathbf{u}}\right|_{\substack{\mathbf{x}=\overline{\mathbf{x}} \\
\mathbf{u}=\mathbf{u}}} .
\end{aligned}
$$

The equilibrium point can be defined as

$$
\overline{\mathbf{x}}=\left[\begin{array}{llllllllllll}
\bar{x} & 0 & \bar{y} & 0 & \bar{z} & 0 & \bar{\psi} & 0 & 0 & 0 & 0 & 0
\end{array}\right]^{T},
$$

at a specific position and velocity

$$
\begin{aligned}
& \overline{\boldsymbol{\Gamma}} \approx\left[\begin{array}{llllll}
\bar{x} & \bar{y} & \bar{z} & \bar{\psi} & 0 & 0
\end{array}\right]^{T}, \\
& \dot{\boldsymbol{\Gamma}} \approx \mathbf{0}_{\mathbf{6} \times \mathbf{1},}
\end{aligned}
$$

where the equilibrium input is

$$
\overline{\mathbf{u}}=\left[\begin{array}{llll}
m g & 0 & 0 & 0
\end{array}\right]^{T} .
$$

The measured outputs are defined as

$$
\mathbf{y}=\left[\begin{array}{llll}
x & y & z & \psi
\end{array}\right]^{T},
$$

and the output matrix $C \in \mathbb{R}^{4 \times 12}$, which is the mapping matrix from $\mathrm{x}$ to $\mathrm{y}$.

In order to use the linearized model (11) as a basis in the design of a discrete-time controller, it is discretized using the zero-order hold method [19], leading to the state-space representation

$$
\mathbf{x}_{k+1}=A_{d} \mathbf{x}_{k}+B_{d} \mathbf{u}_{k}
$$

where the subscript $k$ denotes the current sample. This discretization method depends fundamentally on the selection of a sample time $\delta t$.

TABLE I

QUADROTOR'S PARAMETERS

\begin{tabular}{|c|c|}
\hline Parameter & Value \\
\hline$m$ & $1.568 \mathrm{~kg}$ \\
$L$ & $0.244 \mathrm{~m}$ \\
$J_{x x}$ & $0.0132 \mathrm{~kg} \cdot \mathrm{m}^{2}$ \\
$J_{y y}$ & $0.0116 \mathrm{~kg} \cdot \mathrm{m}^{2}$ \\
$J_{z z}$ & $0.0332 \mathrm{~kg} \cdot \mathrm{m}^{2}$ \\
$k_{M}$ & $0.021 \mathrm{~m}$ \\
\hline
\end{tabular}

Based on the quadrotor's parameters shown in Table I and the linearized model (11), the suitable sample times set $\mathcal{T}_{s}$ can be defined by using the Nyquist-Shannon sampling theorem. According to this theorem, the sampling frequency of a discrete-time system must be at least twice the bandwidth of the continuous-time system. The largest singular value of this quadrotor's model has an analog bandwidth $B_{\sigma}=2.05 \mathrm{~Hz}$, as already established in [20]. Therefore, $\mathcal{T}_{s}$ is defined as

$$
\mathcal{T}_{s}:=\left\{\delta t \mid \delta t \leq \frac{1}{2 B_{\sigma}}\right\}
$$

The specific $\delta t \in \mathcal{T}_{s}$ selected for the discretization of the model is shown in Sections IV-A, IV-B and V, since it must also consider the control law's computation time.

\section{Linear Model Predictive Controller}

The design of MPC systems is based on a mathematical model of the system to be controlled. Control signals are calculated by minimizing an objective function subject to constraints. For this case, an augmented discrete-time statespace quadrotor model is used to design an MPC algorithm that optimizes the control signals and allows trajectorytracking.

\section{A. Formulation of the augmented model}

An augmented model of the system is implemented to add an integral action and to speed-up the OCP solving execution. This augmented model is described as follows.

Let us define the increment variables

$$
\begin{aligned}
\delta \mathbf{x}_{k} & =\mathbf{x}_{k}-\mathbf{x}_{k-1} \\
\delta \mathbf{u}_{k} & =\mathbf{u}_{k}-\mathbf{u}_{k-1}
\end{aligned}
$$

Then, the augmented model is defined as

$$
\begin{aligned}
{\left[\begin{array}{c}
\delta \mathbf{x}_{k+1} \\
\mathbf{y}_{k+1}
\end{array}\right] } & =\left[\begin{array}{cc}
A_{d} & \mathbf{0}_{\mathbf{1 2} \times \mathbf{4}} \\
C A_{d} & \mathcal{I}_{\mathbf{4} \times \mathbf{4}}
\end{array}\right]\left[\begin{array}{c}
\delta \mathbf{x}_{k} \\
\mathbf{y}_{k}
\end{array}\right]+\left[\begin{array}{c}
B_{d} \\
C B_{d}
\end{array}\right] \delta \mathbf{u}_{k} \\
\mathbf{y}_{k} & =\left[\begin{array}{ll}
\mathbf{0}_{\mathbf{4} \times \mathbf{1 2}} & \mathcal{I}_{\mathbf{4} \times \mathbf{4}}
\end{array}\right]\left[\begin{array}{c}
\delta \mathbf{x}_{k} \\
\mathbf{y}_{k}
\end{array}\right]
\end{aligned}
$$

where $\mathcal{I}$ is the identity matrix. For simplicity, we denote the augmented model as

$$
\begin{aligned}
\hat{\mathbf{x}}_{k+1} & =\hat{A} \hat{\mathbf{x}}_{k}+\hat{B} \delta \mathbf{u}_{k} \\
\mathbf{y}_{k} & =\hat{C} \hat{\mathbf{x}}_{k}
\end{aligned}
$$

with $\hat{\mathbf{x}}_{k}=\left[\begin{array}{ll}\delta \mathbf{x}_{k} & \mathbf{y}_{k}\end{array}\right]^{T} \in \mathbb{R}^{16}$.

The reader is referred to [21] for extending the description of the augmented model.

\section{B. Convex optimization problem}

The optimization problem aims to minimize both the tracking error and the control interval signal. The goal is to find an optimal $\delta \mathbf{u}_{k}$ that makes the cost function as small as possible. The benefit of using a cost function in terms of $\delta \mathbf{u}_{k}$ is to avoid large variations and, therefore, to smooth the control signal. This implies that the control signals and the outputs' constraints must be expressed in terms of (22). Thus, the OCP solved at every sample time in the MPC algorithm is given by 


$$
\begin{array}{lll}
\underset{\hat{\mathbf{x}}(\cdot)}{\operatorname{minimize}(\cdot)} & \sum_{k=1}^{N}\left\|\left[\begin{array}{c}
\mathbf{r}_{k}-\mathbf{y}_{k} \\
\delta \mathbf{u}_{k} \\
\hat{\mathbf{x}}_{k}
\end{array}\right]\right\|_{\mathbf{W}}^{2} \\
\text { subject to } & \hat{\mathbf{x}}_{k+1}=\hat{A} \hat{\mathbf{x}}_{k}+\hat{B} \delta \mathbf{u}_{k}, \quad k=1, \ldots, N-1, \\
& \mathbf{y}_{k}=\hat{C} \hat{\mathbf{x}}_{k}, & k=1, \ldots, N, \\
& \delta \mathbf{u}_{\min } \leq \delta \mathbf{u}_{k} \leq \delta \mathbf{u}_{\max }, & k=1, \ldots, N-1, \\
& \mathbf{u}_{\min } \leq \mathbf{u}_{k} \leq \mathbf{u}_{\max }, & k=1, \ldots, N-1, \\
& \hat{\mathbf{x}}_{0}=\mathbf{x}_{\text {est }}, \\
& \hat{\mathbf{x}}_{N} \in \varepsilon
\end{array}
$$

where $\mathbf{r}_{k} \in \mathbb{R}^{4}$ is the reference vector at $k, \mathbf{W} \in \mathbb{R}^{24 \times 24}$ is a positive definite matrix representing the weights in the quadratic cost, $\mathbf{x}_{\text {est }} \in \mathbb{R}^{12}$ is the estimated state vector, $\varepsilon$ is the set of final state constraints, and $\hat{\mathbf{x}}_{k}$ is added to the cost for regularization. The estimated state vector $\mathbf{x}_{e s t}$ is delivered by a Kalman filter, which is executed in the smartphone. The reader is referred to [5] to extend the description of the implemented state estimator.

The OCP in (23) describes a convex optimization problem because its cost function is a convex function and all its constraints are affine.

\section{IMPLEMENTATION RESULTS}

To use a smartphone as a quadrotor flight controller, the control algorithm uses embedded sensors such as inertial measurement unit, global navigation satellite system chipset, and barometer. The smartphone should also be compatible with the Android open accessory protocol. The MPC tests were performed on a LG Nexus 5X smartphone with a Qualcomm Snapdragon 808 hexacore CPU (with a maximum clock rate of $1.8 \mathrm{GHz}$ ), $2 \mathrm{~GB}$ of RAM, and running the Android 8.0 operating system. The sensors in the smartphone are assumed calibrated by Android's sensors application programming interface.

The reference trajectory describes a square in the $x y$-plane, with side-length equal to $3 \mathrm{~m}$, constant altitude of $z=2 \mathrm{~m}$, and flight velocity equal to $0.3 \mathrm{~m} / \mathrm{s}$. The quadrotor dynamics function (10) is also implemented in the smartphone to develop a complete MPC simulation. Both frameworks are tested using a prediction horizon $N=30$.

The frameworks are not compared in terms of number of algorithm's internal iterations, but only with respect to tracking error and total amount of time needed to solve the OCP, here called evaluation time.

\section{A. Implementation with JOptimizer}

The JOptimizer library can handle multivariable functions that require high computational cost by using functions from the Colt library [22], which contains functions of mathematics, linear algebra and multidimensional arrays. These functions are used to define the cost function and the inequality linear constraints. JOptimizer, however, has two main drawbacks in terms of usability in control systems. The first one is that it cannot solve nonconvex problems, which heavily restricts the cost functions and constraints to be used in the OCP. The other drawback is that the solvers embedded in JOptimizer are completely built in Java, which runs on a virtual machine and may introduce delay in the execution of computationally expensive algorithms.

The convex optimization problem (23) is implemented directly in Java using the JOptimizer library and executed in the smartphone. In this case, the sample time used for the discretization of the dynamics is $\delta t=0.050 \mathrm{~s}$, due to the MPC evaluation time shown below. The results of the simulation in the smartphone are shown in Fig. 2 and Fig. 3.

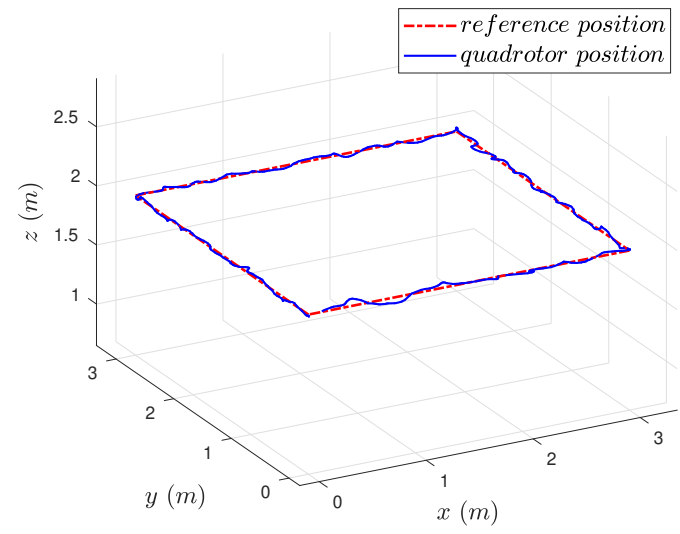

Fig. 2. Trajectory tracking simulation of the MPC solved with JOptimizer.

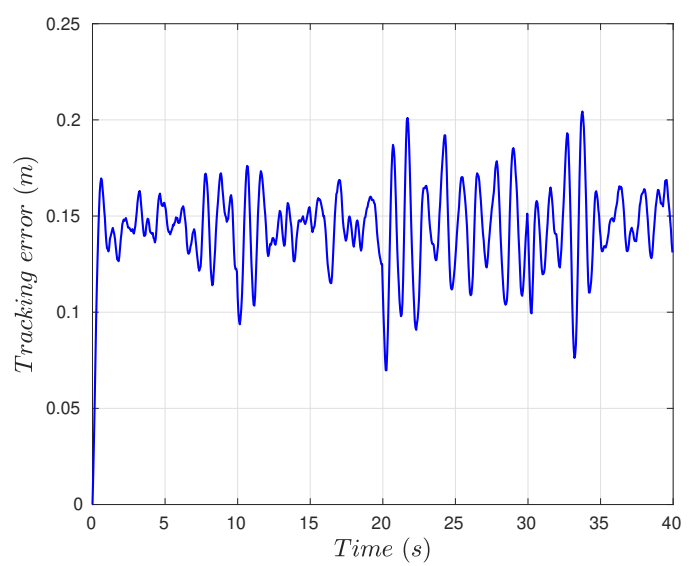

Fig. 3. Tracking error of the MPC solved with JOptimizer.

The execution of the linear MPC with JOptimizer described a mean tracking error of $0.1432 \mathrm{~m}$ with a standard deviation of $0.0226 \mathrm{~m}$. The evaluation time for one iteration of the MPC function, this is one complete solution of the OCP, took in average $40.3573 \mathrm{~ms}$. 


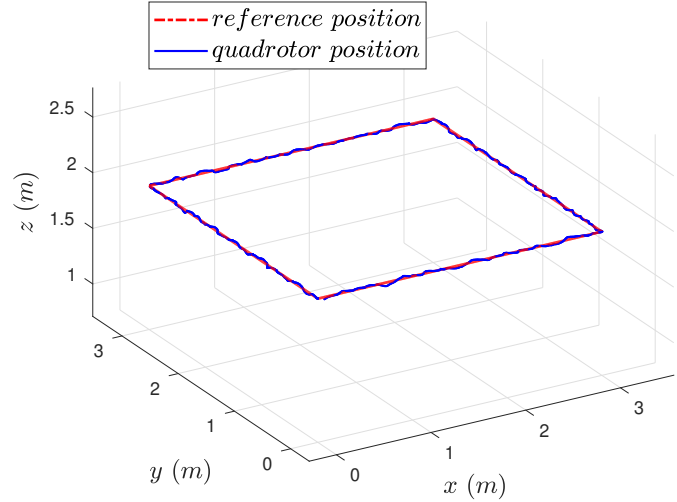

Fig. 4. Trajectory tracking simulation of the MPC solved with CasADi on Android.

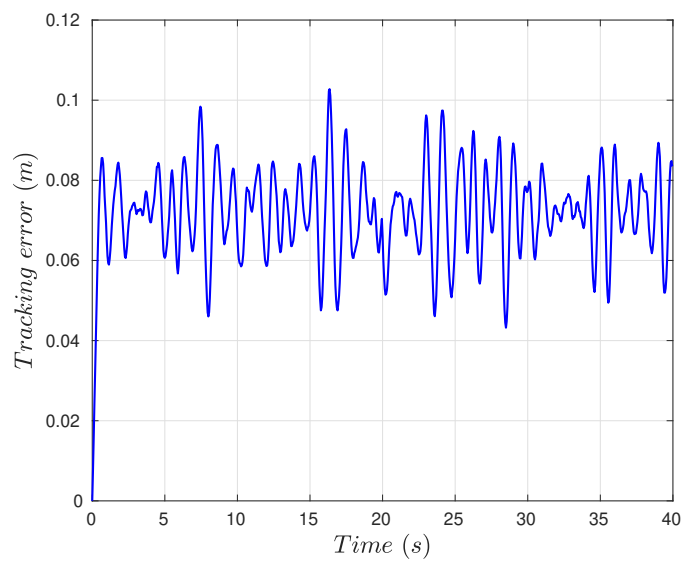

Fig. 5. Tracking error of the MPC solved with CasADi on Android.

\section{B. Implementation with CasADi}

CasADi eases the OCP deployment procedure with the opti stack functions. By using these functions from MATLAB, Python or $\mathrm{C}++$, one can intuitively script an optimization problem and solve it without explicitely programming the algorithm which solves the OCP. On the other hand, there are several numerical solvers interfaced with CasADi, such as IPOPT [23], qpOASES [24], and QRQP [25]. For this implementation we use a sequential quadratic programming approach [26] and QRQP as quadratic program solver.

CasADi is able to generate self-contained $\mathrm{C}$ code for functions such as nonlinear programs, dynamics, integrators, or solvers. For this reason, the convex optimization problem implementation is done in MATLAB using the opti stack, and then code is generated in C. Using JNI, the C code is executed from the Android operating system. For the CasADi-based implementation, the sample time is set to $\delta t=0.010 \mathrm{~s}$. The result of the quadrotor MPC simulation are shown in Fig. 4, while the tracking error is shown in Fig. 5.

In the case of the MPC simulation using CasADi to deploy the OCP, the tracking error has a mean value of $0.0693 \mathrm{~m}$ with a standard deviation of $0.0092 \mathrm{~m}$. The mean evaluation time for MPC simulation on the smartphone using the CasADi framework is $3.8189 \mathrm{~ms}$.

The comparison of the MPC simulation with both the JOptimizer and CasADi frameworks is shown in Table II.

TABLE II

MPC FRAMEWORKS COMPARISON IN ANDROID

\begin{tabular}{|c|c|c|}
\hline Framework & Tracking error & Evaluation time \\
\hline \hline JOptimizer & $0.1432 \mathrm{~m}$ & $40.3573 \mathrm{~ms}$ \\
\hline CasADi & $0.0693 \mathrm{~m}$ & $3.8189 \mathrm{~ms}$ \\
\hline
\end{tabular}

The comparison shows that, using CasADi as deployment framework, the MPC evaluation time and tracking error are reduced by $90.53 \%$ and $51.60 \%$ respectively when compared to the JOptimizer framework. The significant reduction of the evaluation time enables the use of such algorithms within real-time or online implementations of MPC for real systems. The main reason of why the CasADi-based implementation outperforms the JOptimizer one is that CasADi's codegeneration and simplification features speed-up the MPC functions' evaluation during runtime.

\section{Nonlinear Model Predictive Control}

It has been shown that a convex OCP can be fastly solved on Android using CasADi framework to deploy the algorithm. Now the aim is to show that, by using CasADi, it is also possible to solve a nonconvex OCP or a nonlinear MPC on Android. This section shows that, after replacing the linearized dynamics in the OCP (23), the resulting nonconvex OCP can be solved within an MPC fashion in a smartphone.

The nonconvex OCP including the nonlinear dynamics of the quadrotor is defined as

$$
\begin{array}{lll}
\underset{\mathbf{x}(\cdot)) u(\cdot)}{\operatorname{minimize}} & \sum_{k=1}^{N}\left\|\left[\begin{array}{c}
\mathbf{r}_{k}-\mathbf{y}_{k} \\
\mathbf{u}_{k} \\
\mathbf{x}_{k}
\end{array}\right]\right\|_{\mathbf{W}}^{2} & \\
\text { subject to } & \mathbf{x}_{k+1}=\mathcal{R} \mathcal{K}_{4}\left(f\left(\mathbf{x}_{k}, \mathbf{u}_{k}\right), \delta t\right), & k=1, \ldots, N-1, \\
& \mathbf{y}_{k}=C \mathbf{x}_{k} & k=1, \ldots, N \\
& \mathbf{u}_{\min } \leq \mathbf{u}_{k} \leq \mathbf{u}_{\max } & k=1, \ldots, N-1, \\
& \mathbf{x}_{0}=\mathbf{x}_{\text {est }}, & \\
& \mathbf{x}_{N} \in \varepsilon,
\end{array}
$$

where $\mathcal{R K}_{4}$ is the $4 t h$-order Runge-Kutta integrator function applied to equation (10) and it is evaluated in $\left(\mathbf{x}_{k}, \mathbf{u}_{k}\right)$ with $\delta t=0.010 \mathrm{~s}$ and a prediction horizon $N=30$.

This setup was implemented on Android using CasADi code-generation features, the same way as done in Section III. The implementation was tested with the nonlinear dynamics of the quadrotor and had a mean evaluation time of $6.9471 \mathrm{~ms}$. 


\section{CONCLUSION REMARKS AND OUTLOOK}

Online implementation of computationally expensive algorithms, such as model predictive controllers, on smartphones is a big challenge. Especially taking into account that off-the-shelf embedded systems and smartphones have limited computation and instrumentation capabilities. In this paper the use of CasADi as deployment framework for both a linear and a nonlinear model predictive controller for a smartphone-based quadrotor has been presented. Numerical results have shown that the CasADi-based implementation outperforms the one based on JOptimizer, requiring shorter evaluation times and getting lower tracking errors. It is worth investigating the developed controllers performance on a real quadrotor and the use of the real-time iteration scheme to speed-up convergence of the MPC algorithms.

\section{REFERENCES}

[1] J. M. Rodríguez, C. Mateos, and A. Zunino, "Are Smartphones Really Useful for Scientific Computing?" in Advances in New Technologies, Interactive Interfaces and Communicability. ADNTIIC 2011., vol 7547 ed., Springer, Ed., Berlin, 2012, ch. Lecture Notes in Computer Science, pp. 38-47.

[2] D. Martínez, J. Moreno, D. Font, M. Tresanchez, T. Pallejà, M. Teixidó, and J. Palacín, "A Practical Mobile Robot Agent Implementation Based on a Google Android Smartphone," in Distributed Computing and Artificial Intelligence, vol 217 ed., Springer, Ed., 2013, ch. Advances i, pp. 625-632.

[3] M. I. Sani, S. Siregar, M. Z. Irsyad, and Y. I. Wibowo, "Navigation system for smartphone-based autonomous underwater vehicle," in 2017 International Conference on Smart Cities, Automation \& Intelligent Computing Systems (ICON-SONICS). IEEE, nov 2017, pp. 24-29.

[4] G. Loianno, Y. Mulgaonkar, C. Brunner, D. Ahuja, A. Ramanandan, M. Chari, S. Diaz, and V. Kumar, "Smartphones power flying robots," in 2015 IEEE/RSJ International Conference on Intelligent Robots and Systems (IROS). IEEE, sep 2015, pp. 1256-1263.

[5] A. Astudillo, B. Bacca, and E. Rosero, "Optimal and robust controllers design for a smartphone-based quadrotor," in 2017 IEEE 3rd Colombian Conference on Automatic Control (CCAC). IEEE, oct 2017, pp. 1-6.

[6] A. Astudillo, P. Munoz, F. Alvarez, and E. Rosero, "Altitude and attitude cascade controller for a smartphone-based quadcopter," in 2017 International Conference on Unmanned Aircraft Systems (ICUAS). IEEE, jun 2017, pp. 1447-1454.

[7] R. Singhal and P. B. Sujit, "3D trajectory tracking for a quadcopter using MPC on a 3D terrain," in 2015 International Conference on Unmanned Aircraft Systems (ICUAS). IEEE, jun 2015, pp. 1385-1390.

[8] X. Chen and L. Wang, "Cascaded model predictive control of a quadrotor UAV," in 2013 Australian Control Conference. IEEE, nov 2013, pp. 354-359.

[9] H. Cheng and Y. Yang, "Model predictive control and PID for path following of an unmanned quadrotor helicopter," in 2017 12th IEEE Conference on Industrial Electronics and Applications (ICIEA). IEEE, jun 2017, pp. 768-773.

[10] A. Trivellato, "JOptimizer," 2015. [Online]. Available: www.joptimizer.com

[11] K. Chatterjee, H. Fu, A. Goharshady, and N. Okati, "Computational Approaches for Stochastic Shortest Path on Succinct MDPs," in Proceedings of the Twenty-Seventh International Joint Conference on Artificial Intelligence, 2018, pp. 4700-4707.

[12] S. Lee, G. E. Dullerud, and E. Polak, "On the real-time receding horizon control in harbor defense," in 2015 American Control Conference (ACC). IEEE, jul 2015, pp. 3601-3606.

[13] J. A. E. Andersson, J. Gillis, G. Horn, J. B. Rawlings, and M. Diehl, "CasADi: a software framework for nonlinear optimization and optimal control," Mathematical Programming Computation, vol. 11, no. 1, pp. 1-36, 2018.

[14] Y. Zhao, H. Lin, and M. Tomizuka, "Efficient trajectory optimization for robot motion planning," CoRR, vol. abs/1810.04255, 2018.
[15] A. Zanelli, G. Horn, G. Frison, and M. Diehl, "Nonlinear Model Predictive Control of a Human-sized Quadrotor," in 2018 European Control Conference (ECC). IEEE, jun 2018, pp. 1542-1547.

[16] A. Astudillo, L. Garcia, B. Bacca, and E. Rosero, "Model-based Predictive Control for a Smartphone-based Quadrotor," 38th Benelux Meeting on Systems and Control, p. 69, 2019.

[17] M. Faessler, D. Falanga, and D. Scaramuzza, "Thrust Mixing, Saturation, and Body-Rate Control for Accurate Aggressive Quadrotor Flight," IEEE Robotics and Automation Letters, vol. PP, no. 99, pp. 1-7, 2016.

[18] S. Bouabdallah and R. Siegwart, "Backstepping and sliding-mode techniques applied to an indoor micro Quadrotor," Proceedings - IEEE International Conference on Robotics and Automation, vol. 2005, no. April, pp. 2247-2252, 2005.

[19] J. Keller and B. Anderson, "A new approach to the discretization of continuous-time controllers," 1990 American Control Conference, 1990.

[20] A. Astudillo, "Design and Implementation of Flight Dynamics Control Strategies for a Smartphone-based Quadrotor," Universidad del Valle, Tech. Rep., 2017.

[21] M. Abdolhosseini, Y. M. Zhang, and C. A. Rabbath, "An Efficient Model Predictive Control Scheme for an Unmanned Quadrotor Helicopter," Journal of Intelligent \& Robotic Systems, vol. 70, no. 1-4, pp. 27-38, apr 2013.

[22] P. Binko, D. Merlino, W. Hoschek, T. Johnson, and A. Pfeiffer, "The CERN Colt library," 2004. [Online]. Available: https://dst.lbl.gov/ACSSoftware/colt/

[23] A. Wächter and L. T. Biegler, "On the implementation of an interiorpoint filter line-search algorithm for large-scale nonlinear programming," Mathematical Programming, vol. 106, no. 1, pp. 25-57, mar 2006.

[24] H. J. Ferreau, C. Kirches, A. Potschka, H. G. Bock, and M. Diehl, "qpOASES: a parametric active-set algorithm for quadratic programming," Mathematical Programming Computation, vol. 6, no. 4, pp. 327-363, dec 2014.

[25] J. A. E. Andersson and J. B. Rawlings, "Sensitivity Analysis for Nonlinear Programming in CasADi," IFAC-PapersOnLine, vol. 51, no. 20, pp. 331-336, 2018.

[26] J. De O. Pantoja and D. Mayne, "A sequential quadratic programming algorithm for discrete optimal control problems with control inequality constraints," in Proceedings of the 28th IEEE Conference on Decision and Control. IEEE, pp. 353-357. 